FERMILAB- TM-2696-DI

October 2018

\title{
Accelerator/Experiment Operations - FY 2018
}

\author{
G. Bock ${ }^{1}$, M. Convery, K. Decker ${ }^{2}$, L. Fields, B.T. Fleming, D. Harris, D. Johnson, T. Kobilarcik, M. \\ Lancaster, D. Newhart, C. Polly, M. Rominsky, J.J. Schmidt, \\ P. Shanahan, P. Vahle, G.P. Zeller
}

\section{Introduction}

This Technical Memorandum summarizes the Fermilab accelerator and experiment operations for FY 2018. It is one of a series of annual publications intended to gather information in one place. In this case, the information concerns the FY 2018 NOvA and MINERvA experiments using the Main Injector Neutrino Beam (NuMI), the MicroBooNE experiment, LArIAT experiment, and Meson Test Beam activities in the $120 \mathrm{GeV}$ external switchyard beam (SY120).

Each section was prepared by the relevant authors, and was then edited for inclusion in this summary.

Accelerator Operations (M. Convery, D. Johnson, T. Kobilarcik, D. Newhart)

The low-energy and high-energy neutrino beams, muon beam to g-2, and the test beams in the Meson area were brought into operation after the summer 2017 accelerator shutdown. The Booster Neutrino Beam startup occurred on October 27, beam to MTest began on November 10, and NuMI beam started up on November 16. Commissioning beam to the g-2 experiment resumed on November 17 and the experiment started collecting physics data on March 22. Beam was delivered to users until the present shutdown which began July 7.

The high-energy neutrino beam was scheduled for beam delivery for 37 weeks. NuMI beam power ramped up quickly to $700 \mathrm{~kW}$ after the shutdown. There were 5026 hours of high-energy operation in FY 2018 with $5.7 \times 10^{20}$ protons delivered. Integrated beam to NuMI met expectations for the year.

The Booster Neutrino Beam exceeded the design goal for FY2018 due to delays in g-2 readiness for physics. About 5454 hours of beam were and $3.1 \times 10^{20}$ protons were delivered to the MicroBooNE experiment.

For the Muon Campus, the PEMP notable of 5000 muons/sec stored in the g-2 ring was achieved on January 12. By the time the experiment was ready for physics data in March, the accelerator was ready for operation at full repetition rate and intensity of protons on target. Since March 22, 1701 hours of beam were delivered to the g-2 experiment.

${ }^{1}$ Editor

${ }^{2}$ Administrative Support 
The Fermilab Test Beam Facility was scheduled for beam for 35 weeks of the 52-week period. The facility operated for 2290 hours driven by user requests. Causes of broken wires and vacuum problems in the MI extraction septa which caused significant downtime in 2017 were addressed by a task force with success in resolving the vacuum issues. A few days were lost due to a broken wire in December, and the test beam was down for roughly two weeks in February when a dipole magnet in the Meson beamline developed a cooling water leak and was replaced.

In MCenter, the LArIAT (PixLAr) experiment restarted operation in December and took beam for 10 weeks, non-consecutive. During those weeks, over 722 hours, $4.0 \times 10^{13}$ protons were delivered to the experiment. The SBN Cold electronics experiment also received $3.2 \times 10^{12}$ protons in 71 hours over two weeks at the end of June.

The length of the 2018 summer shutdown was driven by maintenance of cooling water systems throughout the complex. Other important work included completion of the Marx modulators for the Linac RF, continued M4 beamline installation for Mu2e in the enclosure shared with the M5 line to g-2, and the installation of sextupoles for resonance compensation in the Recycler.

The following plots summarize the beam delivery in FY 2018. Note that the plot of beam to g-2 begins at the time when $\mathrm{g}-2$ began taking physics data. The goals were redefined to start from that point, but with the proton flux (red/blue-line slope) as projected in the original design/base goals.

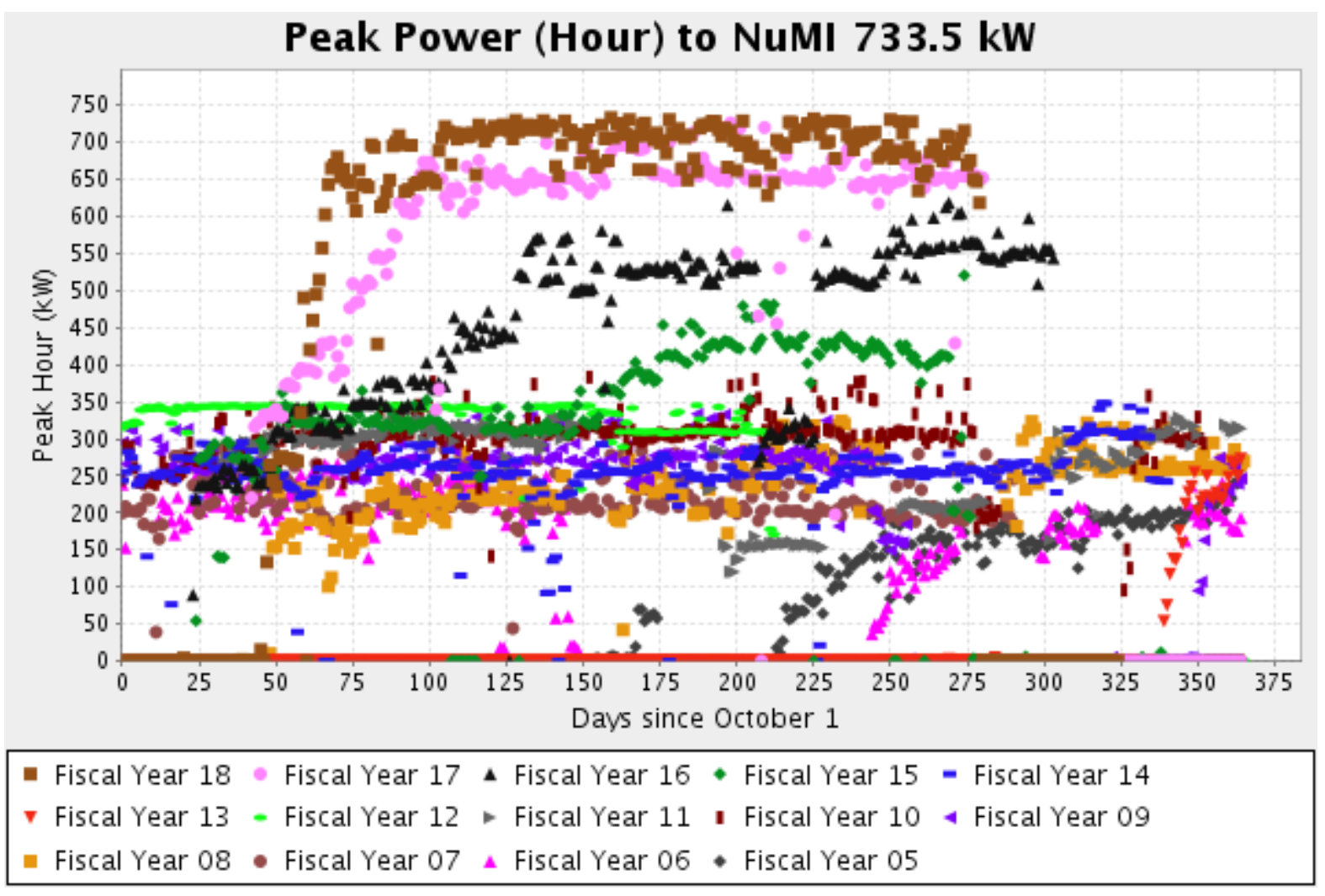



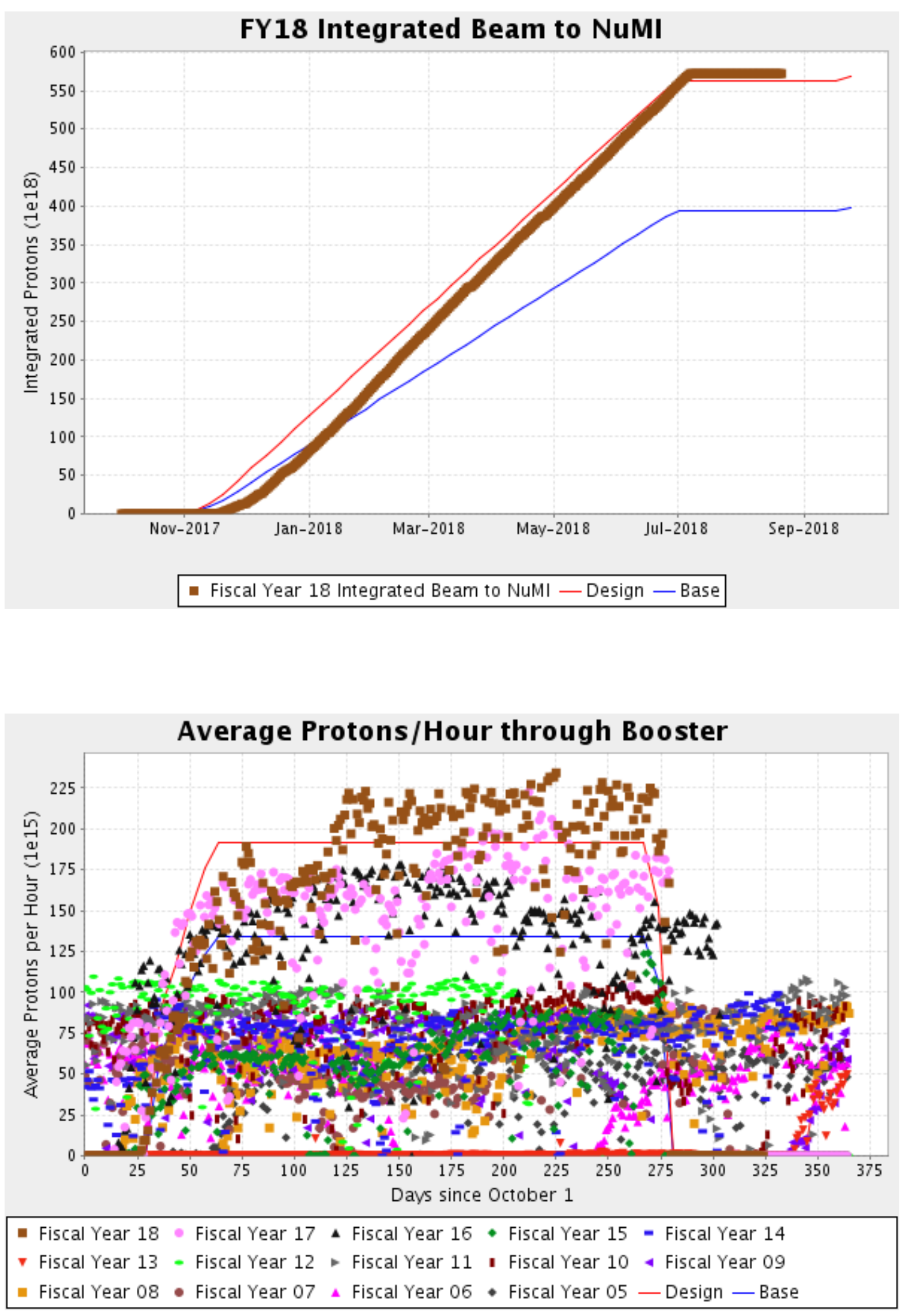


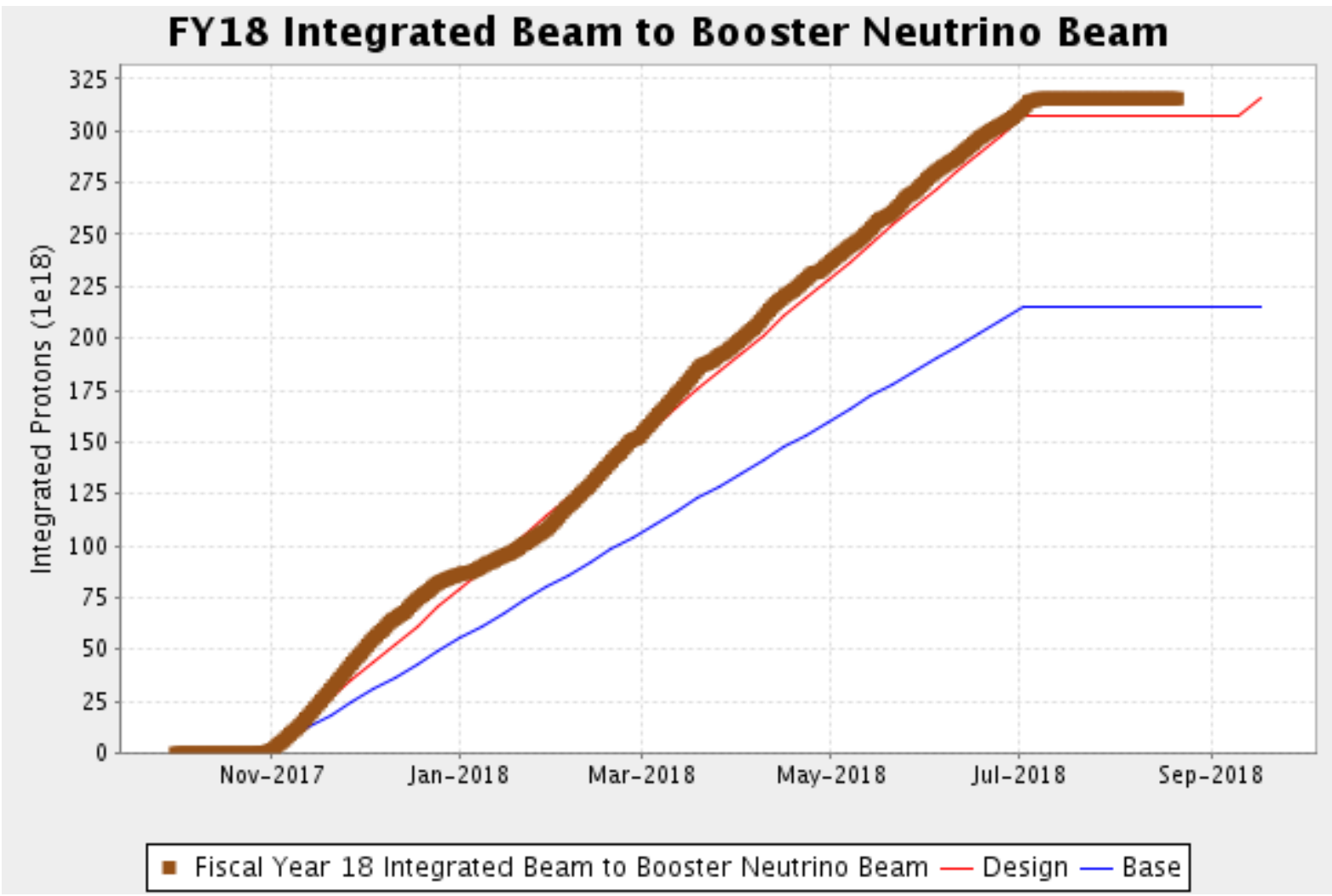

FY18 Integrated Beam to Muon

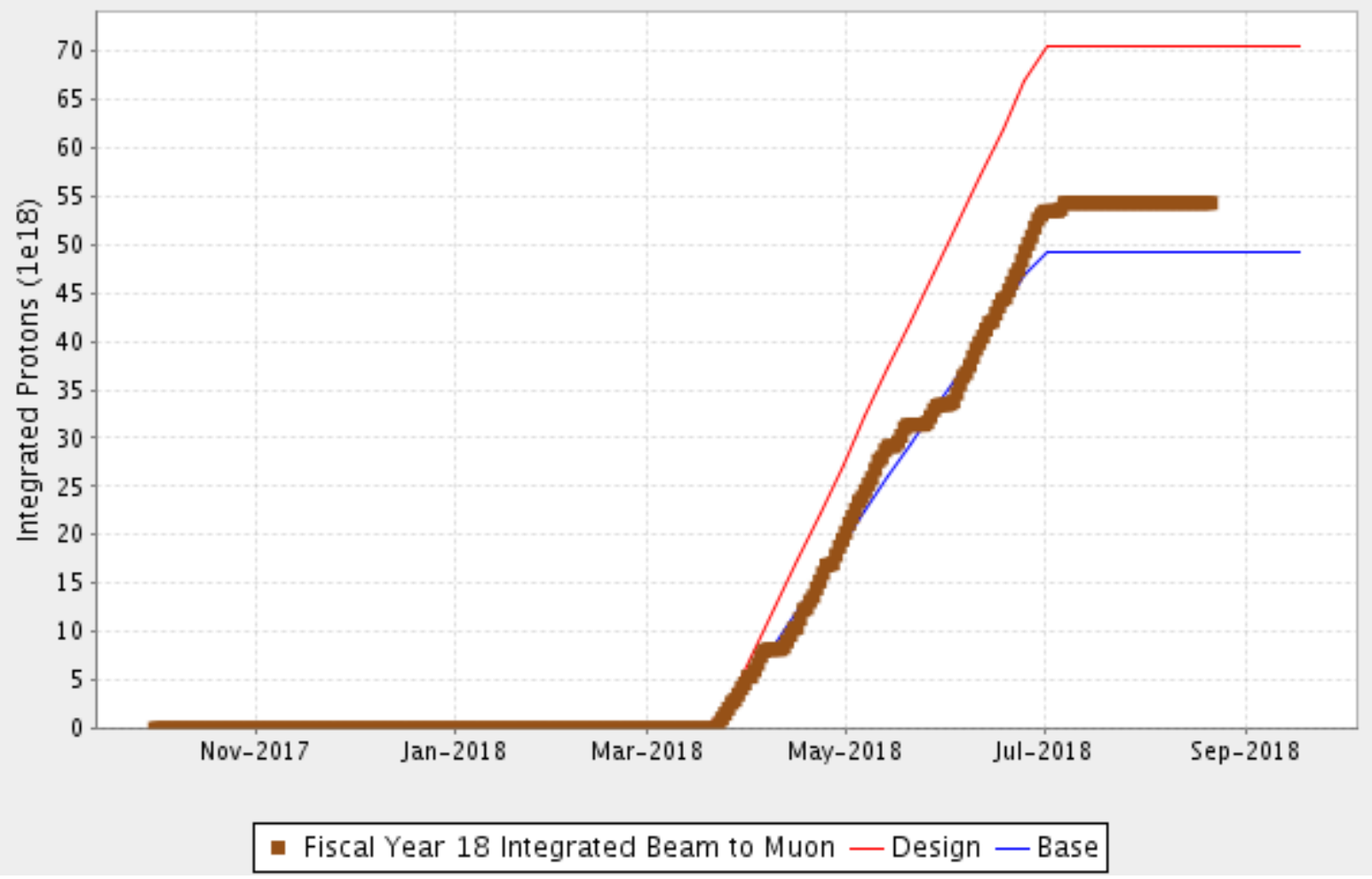




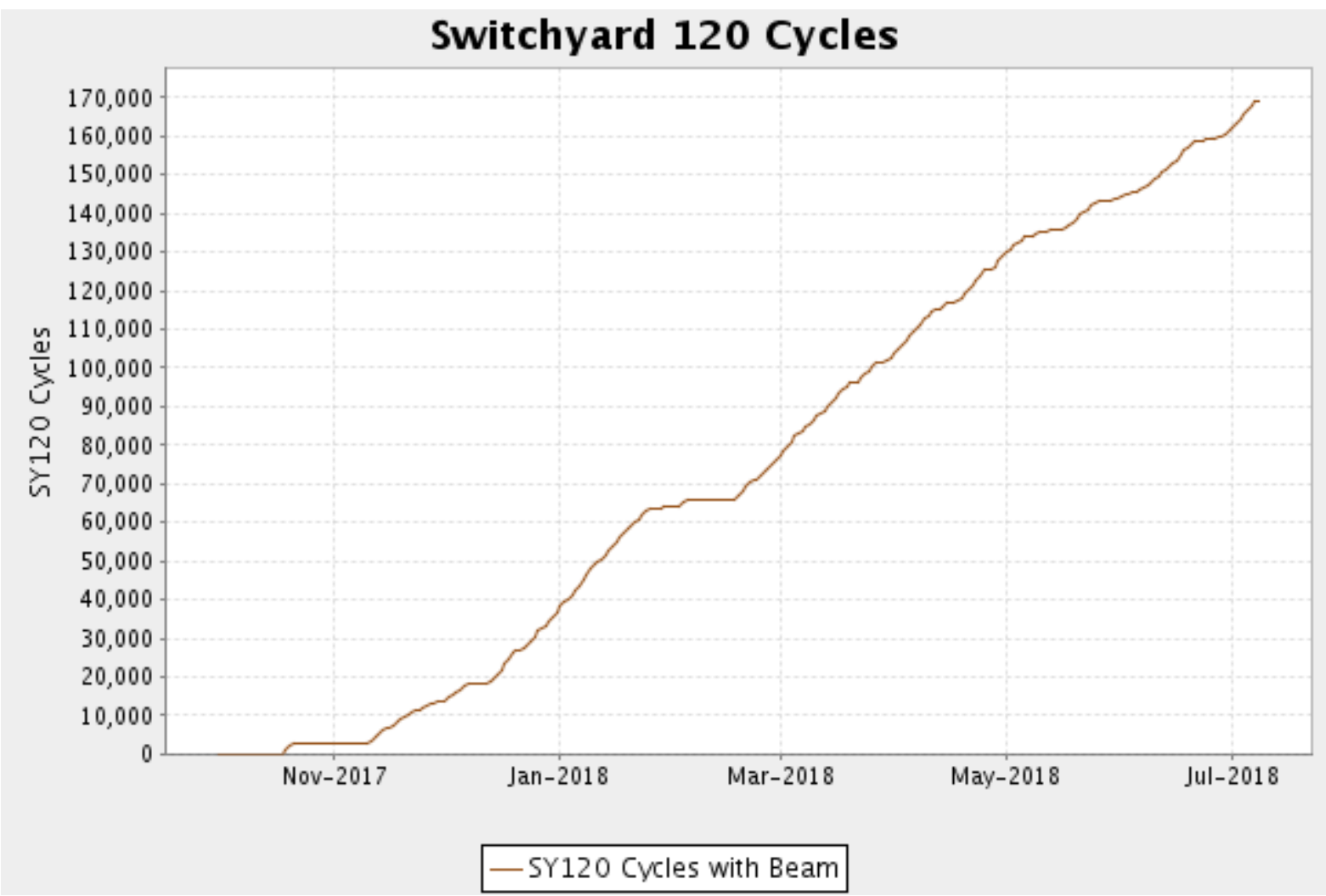

NOvA Report (P. Vahle and P. Shanahan)

NOvA completed its fourth year of operations in FY 2018. The experiment recorded $5.6 \times 10^{20}$ protons-on-target (POT) this fiscal year, all in antineutrino mode. To date, NOvA has accumulated a NuMI beam exposure in its Far Detector of $8.9 \times 10^{20}$ protons-on-target (POT) (full-detector-equivalent, accounting for beam delivered when the detector was under construction) with NuMI operating in neutrino mode, and $9.4 \times 10^{20}$ POT with NuMI operating in antineutrino mode. The NOvA collaboration presented two new sets of long-baseline oscillation results, including its first antineutrino results showing the first strong evidence for long-baseline electron antineutrino appearance. NOvA also presented its first neutrino interaction crosssection results, and published two papers in PRD. In addition to analysis activity and operations of its near and far detectors, the experiment began installation of a detector and instrumentation for a test beam.

NOvA research resulted in 8 Ph.D.'s being awarded in FY 2018, bringing the total to-date to 23. Two institutions - Syracuse and U. Wisconsin, Madison - joined NOvA in FY18. Winona State left the collaboration, bringing the total number of collaborating institutions to 49. Unfortunately, ongoing uncertainty in the funding situation of the 9 Indian institutions on NOvA has left their ability to continue to participate in the collaboration in doubt.

The experiment continued to staff data-taking shifts 24 hours per day without exception, with experts in data acquisition, detector controls, power supplies, and avalanche photo diode (APD) operations on-call $24 \times 7$. Shifts took place at one of 19 Remote Operations Centers (ROC) 
including the ROC-West at Fermilab. A new ROC at Syracuse U. was commissioned. A team of 3 Run Coordinators serving in a rotation oversaw daily operations and coordinated activities of the shifters, experts, and support staff. The maintenance and operation of the far detector, and of the Ash River Laboratory, were performed by a crew employed by the University of Minnesota under budget and safety oversight from Fermilab, and in coordination with the collaboration operations team.

The uptime of both detectors was generally high, with the Far Detector being live for $98.7 \%$ of protons delivered to the NuMI target during FY18. Relatively little maintenance was required. Only 105 Front End Board/Thermal Electric Cooler Controller (FEB/TECC) units out of 10752 were swapped during FY 2018. More than 1000 good spares remain, and no effort has yet been made to diagnose and repair the ones that have been removed. Thirty-seven avalanche photodiodes (APD) were swapped; 69 assembled APDs are in reserve for the Far Detector and about 600 bare APDs remain. All but about $0.06 \%$ of the FEB/TECCs are typically active during data-taking. No swaps of DCMs or power distribution components were made this year.

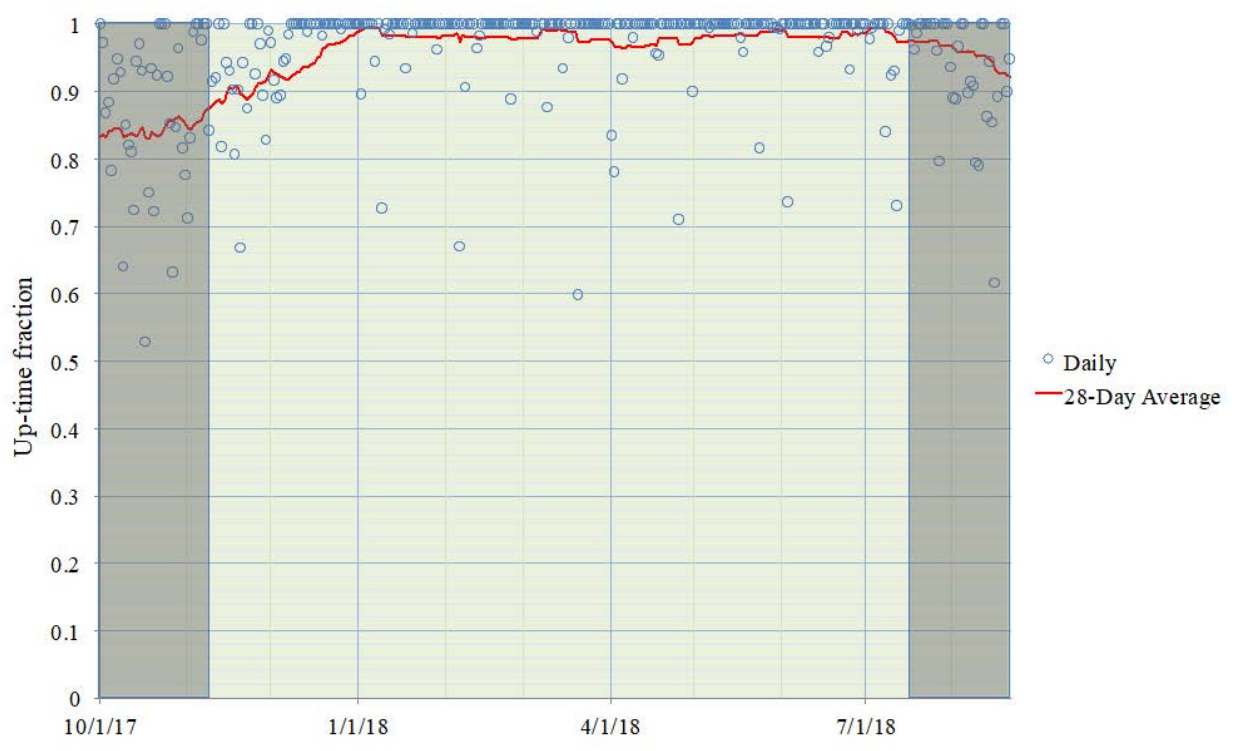

Figure N-1: The NOvA far detector uptime in FY18. This shows the daily (points) and 28-day (red line) average uptime of the NOvA detector systems including planned downtimes for maintenance during beam-off times. Accelerator shutdown periods are indicated by the shaded regions. Overall the detector operated $97 \%$ of the time during FY18, with beam-weighted uptime of 98.7\%. 


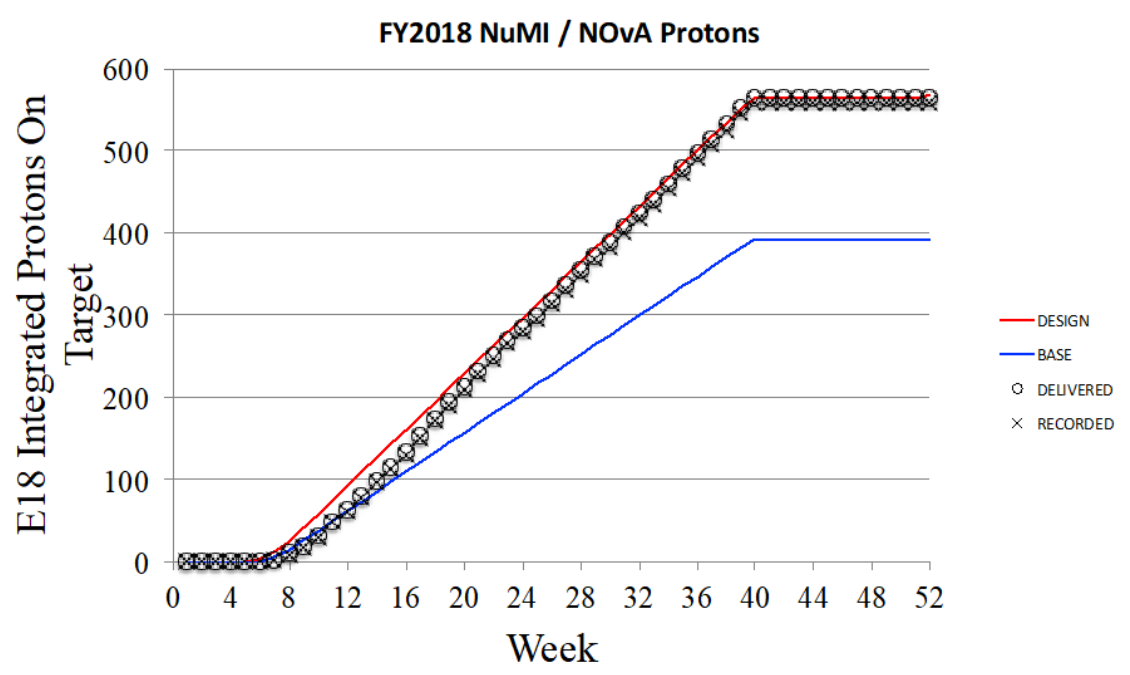

Figure N-2: The total protons on target delivered and recorded by the NOvA far detector in FY18 vs week. The far detector recorded data for 5.6E20 of the 5.7E20 protons delivered to the NuMI target (98.7\%). The NuMI beam was operated in anti-neutrino mode for the full fiscal year.

The near detector had 99.6\% beam-weighted uptime for FY18. Eight FEBs were swapped in FY 2018. No APDs were swapped. There are adequate ND spares, with 18 assembled APDs and 31 FEBs in reserve.

Figures N-1 and N-2 show the detector uptimes and delivery of protons to NuMI for FY 2018.

In FY2018, NOvA released results from the analysis of an 8.85e20 protons-on-target exposure of neutrino data, the full data set recorded in neutrino mode to date. This analysis featured an improved detector light model, updated tuning of the neutrino interaction model, and enhanced sensitivity to maximal mixing by separating $v_{\mu}$ events into energy resolution bins. A fit to both disappearance and appearance channels favors the normal mass hierarchy with values of $\Delta \mathrm{m}^{2} 32$ from $(2.37-2.52) \times 10^{-3} \mathrm{eV}^{2}, \sin ^{2}\left(\theta_{23}\right)$ from $(0.43-0.51)$ or $(0.52-0.60)$, and $\delta_{\mathrm{CP}}$ from $(0,0.12 \pi)$ or $(0.91 \pi, 2 \pi)$ allowed at the $68 \%$ confidence level. These results have been published in PRD 98, 032012 (2018). The paper was featured as an Editors' Suggestion, a paper judged to be "particularly important, interesting, and well written."

NOvA also released results from its first antineutrino data set of $6.9 \mathrm{e} 20$ protons-on-target in FY2018. The full power of the NOvA physics program comes from comparing oscillations between neutrinos and antineutrinos. The experiment sees $4 \sigma$ evidence of electron antineutrino appearance, the first ever strong evidence of this transition occurring over a long-baseline. A combined fit to all NOvA data prefers the normal mass hierarchy at $1.8 \sigma$. The best fit is in the upper octant, and the data disfavor maximal mu-tau flavor mixing in the third neutrino mass state also at $1.8 \sigma$. Values of $\delta_{\mathrm{CP}}$ near $\pi / 2$ in the inverted mass hierarchy are disfavored at more than $3 \sigma$. Figure N-3 shows the allowed region in $\theta_{23}-\delta_{\text {CP }}$ parameter space coming from the combined fit of appearance and disappearance data in both neutrino and antineutrino mode. 


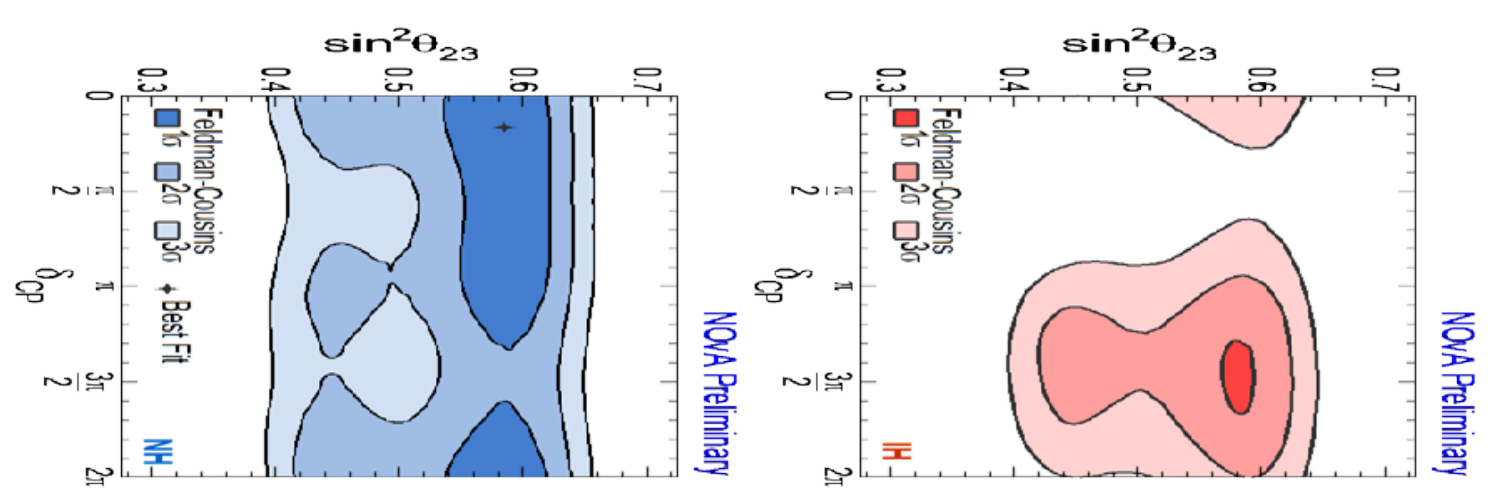

Figure N-3: The significance contours in $\sin ^{2}\left(\theta_{23}\right), \delta_{\mathrm{CP}}$, and the Mass Hierarchy (Left: Normal, Right: Inverted) for the combined NOvA muon neutrino disappearance and electron neutrino appearance fit using $\theta_{13}$ from reactor neutrino disappearance. Unshaded regions are excluded at more than 3 sigma.

In addition to oscillation measurements, NOvA also released first cross section measurements in a Joint Experimental Theoretical Physics seminar at Fermilab in Dec. 2017. This talk featured the measurement of both neutral current coherent $\pi^{0}$ production and charged current semiinclusive $\pi^{0}$ production. Both measurements support the oscillation program; coherent $\pi^{0}$ production is a background to $v_{\mathrm{e}}$ appearance, while charged current $\pi^{0}$ production is sensitive to nuclear effects that could affect energy reconstruction. Beyond the impact on oscillation analyses, measuring these processes is of interest to the broader neutrino interaction community. In October, NOvA published its first sterile neutrino search result, originally presented in 2016, in Phys. Rev. D 96, 072006.

In FY2018, the NOvA collaboration began construction and commissioning of a testbeam detector to run in the Fermilab Test Beam Facility. When data taking starts early in calendar year 2019, the detector will collect data from tagged electron, muon, pion, and proton beams which will enable a detailed understanding of the detector's muon energy scale, electromagnetic and hadronic response in addition to providing real data for the detailed study of particle identification techniques.

The NOvA collaboration has identified several potential improvements in analysis and beam operations to enhance the physics reach of the experiment. Accelerator Improvement Projects, including a new target design, improvements to the target area, and measures to reduce losses in the Booster, together enable up to $900 \mathrm{~kW}$ beam power to be delived to NOvA by FY2021. For favorable combinations of oscillation parameters currently favored by world data, these improvements bring 3 sigma sensitivity to resolution of the mass hierarchy within reach in 2020. Greater than 5 sigma sensitvity is possible by the end of the run in 2024 for these favorable parameters.

E-938 / MINERvA Report (L. Fields, D. Harris)

The MINERvA experiment took physics quality data in in the NuMI Low Energy beam from November 2009 until summer 2013. In FY 2014 through FY 2017, the MINERvA experiment took data in the NuMI Medium Energy beam, and continued this data taking in FY 2018. 


\section{FY 2018 Operations}

The data taken in FY 2018 was all Medium Energy antineutrino data $(6 \mathrm{GeV}$ peak antineutrino energy). Between October 1, 2017 and July 7, 2018, the experiment received 5.72×10 ${ }^{20}$ POT in antineutrino mode. Since 2013 the experiment has received $21.5 \times 10^{20} \mathrm{POT}$ in medium energy running, taken with a MINERvA Detector integrated livetime of $97.0 \%$.

During the FY 2018 running period, the collaboration continued to calibrate the detector, monitor the detector light levels, and check the reconstruction performance of both the MINERvA and MINOS detectors continuously. The experiment operates $40 \%$ of its shifts remotely, and in FY 2018 continued its checklist-based shifts. Figure MV-1 shows the FY 2018 protons delivered and recorded versus time, including the POT recorded when the MINERvA detector was live and, noting that some MINERvA analyses use the MINOS near detector, the POT recorded when both MINERvA and the MINOS Near Detector were live. In FY 2017, MINERvA took data with helium in the cryostat and water in the water target. The MINERvA collaboration took responsibility for the MINOS Near Detector in Spring 2016, including data processing, data quality monitoring, and routine swaps of MINOS electronics boards in case of failures.



Figure MV-1: Shows the protons on target versus time, as well as the integrated livetimes of the MINERvA detector alone and the MINERvA and MINOS Near detector combined for FY18. 
MINER vA Construction and Installation Activities in FY 2018

During FY18 MINERvA did not change anything associated with its DAQ or electronics. During the spring of 2018 the Helium Target was emptied in preparation for taking Helium Target Empty data so that a background subtraction could be done for a Medium Energy Antineutrino Helium Analysis.

\section{MINER vA Results through FY 2018}

The low-energy neutrino data, which is still being analyzed, was taken to provide exclusive cross-section measurements. Low energy events tend to have few final state particles, which allows the identification of single particles in the MINERvA detector and the measurement of exclusive channels important for current and future oscillation experiments. The MINERvA detector has a granularity that is about a factor of 10 more than the NOvA detector, and can be used to identify processes that will contribute backgrounds and signal processes in NOvA, DUNE, T2K and the SBN experiments.

By the start of 2018, the MINERvA Collaboration had published a total of 20 papers covering a broad range of neutrino and antineutrino interaction channels. By FY16 the channels included different ways of analyzing the charged current quasi-elastic interaction, a measurement of the inclusive charged current cross section ratios lead/scintillator, iron/scintillator, and carbon/scintillator, neutrino and antineutrino production of charged and neutral pions, coherent production of charged pions by both neutrinos and antineutrinos, and a first electron neutrino charged current quasi-elastic cross section measurement. The FY17 publications include measurements of both charged current inclusive and charged current coherent kaon production, diffractive neutral pion production, and deep inelastic scattering cross section ratios on carbon, iron and lead to scintillator.

In FY18 MINERvA published five additional cross section results, all from our low energy data set. These results include our measurement of single neutral pion production by neutrinos [Phys Rev D 96, 072003 (2017)], our antineutrino double differential charged current quasi-elastic-like cross section as a function of muon kinematics [Phys Rev D 97, 052002 (2018)], our neutrino and antineutrino charged current coherent differential cross sections as a function of pion and muon kinematics [Phys Rev D 97, 032014 (2018)], and two Physical Review Letter publications, one on antineutrino low recoil events [Phys Rev Lett., 120221805 (2018)] and one on transverse variables in events with a proton, muon, and no pions [Phys Rev Lett 121, 022504 (2018)], both of which will be described in more detail below.

MINERvA has also made significant progress with analysis and simulation of the medium energy neutrino data set. In FY17 we completely overhauled the way we simulated accidental activity in the detector as a function of booster batch intensity, and in FY18 we carried out a successful processing campaign where we simulated 2 times our neutrino data statistics in the scintillator tracker and 10 times our neutrino data statistics in the nuclear target region where our analyses are more likely to be statistics limited. We are currently in the process of finalizing our first medium energy analyses and expect to start publishing Medium Energy data in the next six months. We have also started making plans for a data preservation campaign that will enable future analyses of our data, and/or enable future models to be tested against our data. 

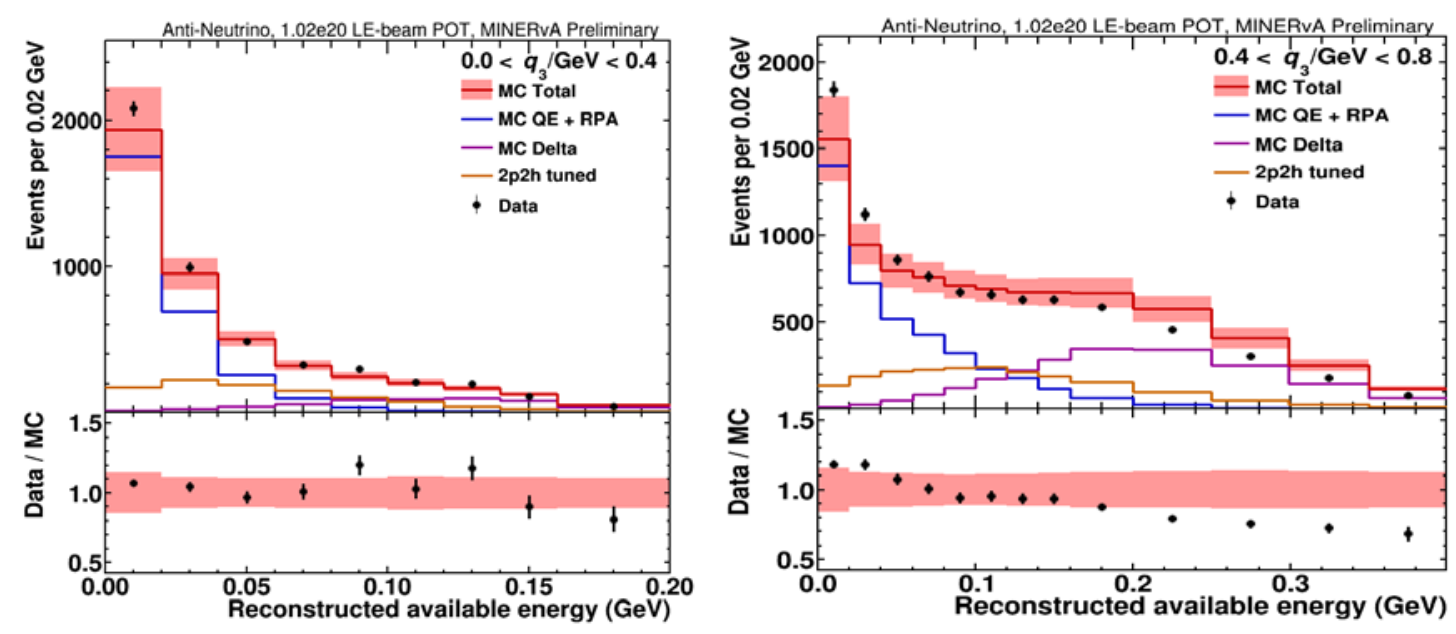

Figure 2 shows the event distributions as a function of available energy (defined in the text) for antineutrino charged current events with 3-momentum transfer below $0.4 \mathrm{GeV}$ on the left, and between 0.4 and $0.8 \mathrm{GeV}$ on the right.

MINERvA's measurement of low recoil events in neutrino interactions made a huge impact in the oscillation community because it was the first time that multi-nucleon scattering events were cleanly identified in neutrino interactions in a way that tests the hadronic predictions of models of those events. The technique is to measure cross sections in the 2-dimensional plane of available energy (hadronic energy that is visible in a scintillator detector) transferred versus 3momentum transferred (as estimated by both muon and hadronic recoil information). This technique has been adopted by NOvA and is being considered by T2K, and in the meantime a tune based on that analysis has been used by NOvA and T2K to help evaluate their uncertainties associated with imperfect knowledge of that process in those experiments. In FY18 MINERvA published a similar analysis in Low Energy antineutrino interactions with low recoil. This analysis showed evidence of multi-nucleon interactions in antineutrino scattering, and in fact the tune that was produced from the neutrino analysis better reproduces the antineutrino results, as shown in Figure 2 above, than the default GENIE simulation of these events [PRL, 120221805 (2018).]

This year MINERvA also demonstrated a new way to untangle the effect of the nucleus in neutrino interactions by using the kinematics of both the outgoing proton and muon in pion-less charged current interactions. By selecting protons that are well-reconstructed in the MINERvA detector for events with a well-reconstructed muon and proton, several new observables become available to shed light on nuclear effects. In particular, the initial neutron momentum ( $\mathrm{p}_{\mathrm{n}}$, usually assumed to be zero or at least less than the "Fermi momentum") can be calculated, as well as the momentum imbalance in the plane transverse to the neutrino direction. The direction of that momentum imbalance, defined as $\alpha_{\mathrm{T}}$, should be evenly distributed from 0 to 180 degrees if the only thing occurring in the nucleus is Fermi motion. Multinucleon correlations and final state interactions (FSI) will each modify those observables, but in different ways. This can be seen in the figures below. Again, the current model that MINERvA has produced by tuning completely different observables for a broader event sample seems to reproduce these new variables much better than a prediction that does not have this extra strength in multinucleon effects [Phys Rev Lett 121, 022504 (2018)]. 

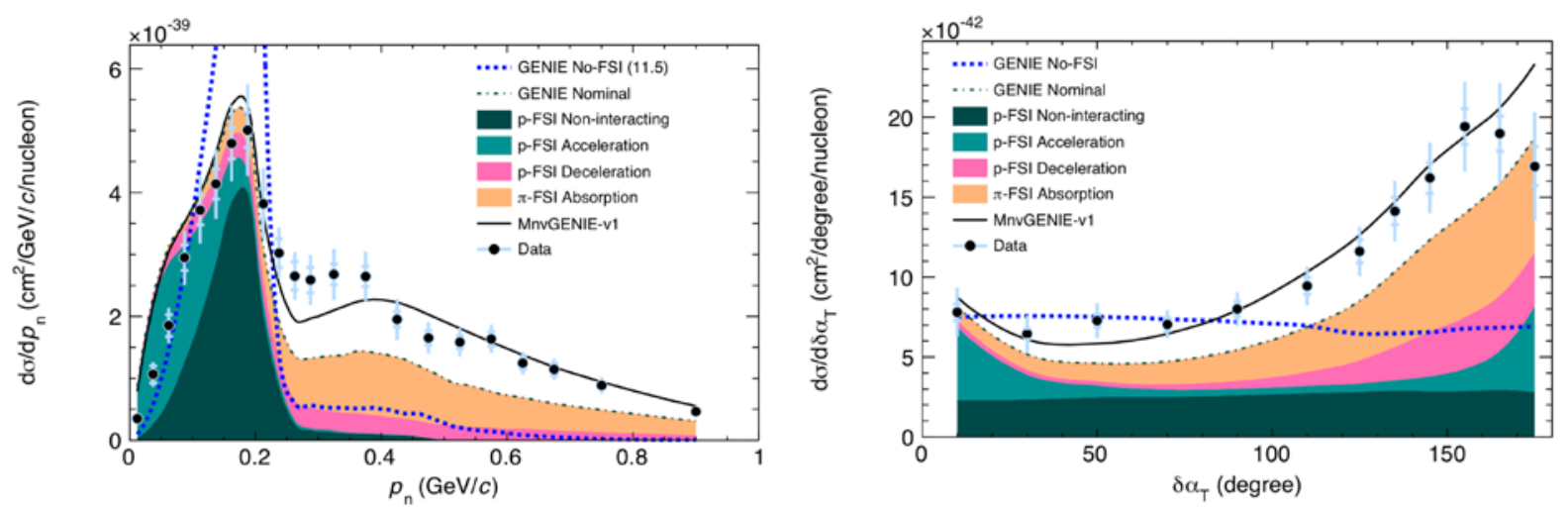

Figure 3 (left) shows the differential cross section as a function of the initial state neutron momentum. The figure at the right shows the cross section as a function of the direction of the transverse momentum imbalance in muon plus proton events ( $\alpha_{T}$, defined in the text). The additional strength indicated between the GENIE Nominal and the black line comes from a fit to MINERVA's low recoil data as a function of 3-momentum transferred and available energy.

Fixed Target Switchyard $120 \mathrm{GeV}$ (SY120) and MTest Reports (T. Kobilarcik, M. Rominsky, J.J. Schmidt)

The general operation of SY120 was relatively smooth. The MTest beamline was operated for the FTBF program as requested by the test beam experiments. The MCenter beamline, with tertiary beam capability, primarily supported the LArIAT experiment, although other test beam experiments, as part of the FTBF program, were also supported. Instrumentation improvements continued to be developed, with emphasis on improving the beam position and beam intensity systems. During the FY 2018 shutdown, final modifications to the primary beam vacuum system were completed.

The Fermilab Test Beam Facility (M. Rominsky, J.J. Schmidt)

The Fermilab Test Beam Facility (FTBF) provides users from around the world an opportunity to test detectors in a variety of charged particle beams. The facility offers two beamlines (MTest and MCenter) to accomplish this goal. A plan view of the facility is shown in Fig. TB-1. The facility offers instrumentation to understand the beamline and infrastructure such as gas lines, high voltage lines, and signal cables. 


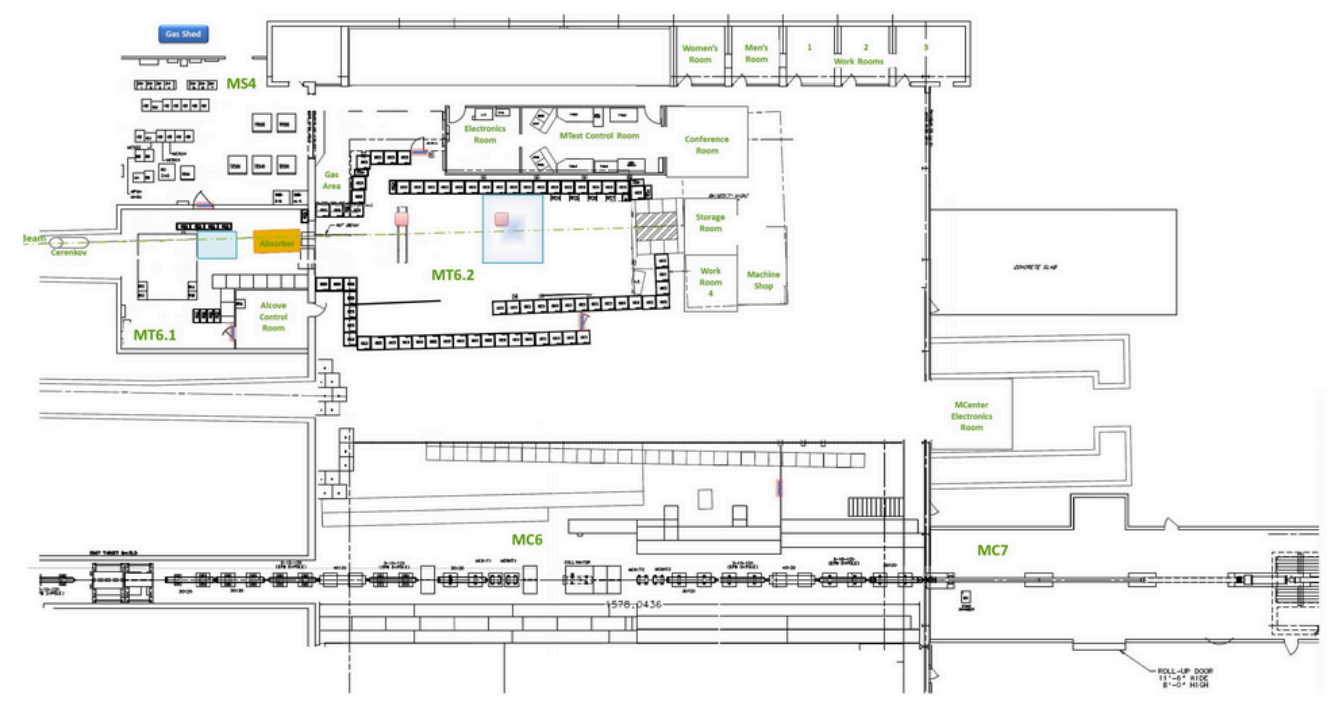

Figure TB-1: View of the Fermilab Test Beam Facility. The beamline at the top of the picture is MTest and the beamline at the bottom of the picture is MCenter.

Research Performed at the FTBF in FY 2018

Each test beam experiment is required to prepare a Technical Scope of Work (TSW) with the laboratory in which the beam, infrastructure, and safety requirements are spelled out in detail. Four new experiments were approved in FY 2018 and started taking data. Seventeen experiments returned to continue their programs. These 21 experiments are listed in Table TB- 1 and represent 216 collaborators at instituions all over the world. Figure TB-2 shows how the experiments broke down by research focus. We supported groups from both ATLAS and CMS, as well as groups from Brookhaven and the Relativistic Heavy Ion Collider (RHIC). We supported the neutrino experiments LArIAT and SBN Cold Electronics. We also had several groups come in to test general detector research and development. Full information from these groups will be included in the annual test beam report (in preparation).

The experiments fully utilized the MTest beamline for data taking purposes out of the 34 weeks that were available with beam during FY18. Counting each user per week yields 54 weeks of experiment time during FY 2018, with multiple users in the beam line at the same time. The MCenter beamline was used for data taking for the LArIAT experiment (T1034) and the SBND Cold Electronics tests. 


\begin{tabular}{|c|c|}
\hline Experiment Number & Description \\
\hline T1041 & CMS Forward Calorimetry R\&D \\
\hline T0992 & Fermilab FCP130 \\
\hline T0992 & US CMS Pixels Phase II Sensors \\
\hline T0992 & INFN CMS Pixels Phase II Sensors \\
\hline T0992 & CMS Outer Tracker Phase 2 \\
\hline T0992 & CMS RD53a Chip \\
\hline T1209 & CMS Outer Tracker R\&D \\
\hline T1441 & sPHENIX MAPS Vertex Detector (MVTX) \\
\hline T1068 & Beam Tests of the SVX4 Telescope \\
\hline T1396 & $\begin{array}{l}\text { EMPHATIC (Hadron Emulsion Detectors for } \\
\text { Neutrinos) }\end{array}$ \\
\hline T1044 & sPHENIX Calorimetry Tests \\
\hline T1224 & ATLAS Pixel Telescope Tests \\
\hline T1429 & $\begin{array}{l}\text { Performance Study of a planar GEM and MM } \\
\text { detector with zigzag pad readout }\end{array}$ \\
\hline T1439 & sPHENIX Silicon Strip Tracker (INTT) Testsu \\
\hline EDIT & $\begin{array}{l}\text { Excellence in Detector and Instrumentation } \\
\text { Techniques School }\end{array}$ \\
\hline T1443 & SBND Vertical Slice Test \\
\hline T1473 & $\begin{array}{l}\text { FLYSUB-Consortium Tracking and RICH } \\
\text { Performance Evaluation }\end{array}$ \\
\hline T1272 & EM Mini Calorimeter \\
\hline T1450 & EIC PID R\&D: Argonne MCP-PMT Test \\
\hline T1034 & LArIAT: Liquid Argon in a Test Beam \\
\hline T1297 - 02 & PixLAr \\
\hline
\end{tabular}

Table TB-1: Test Beam experiments performed in FY 2018. 


\section{FY18 USER GROUP BY RESEARCH FOCUS}

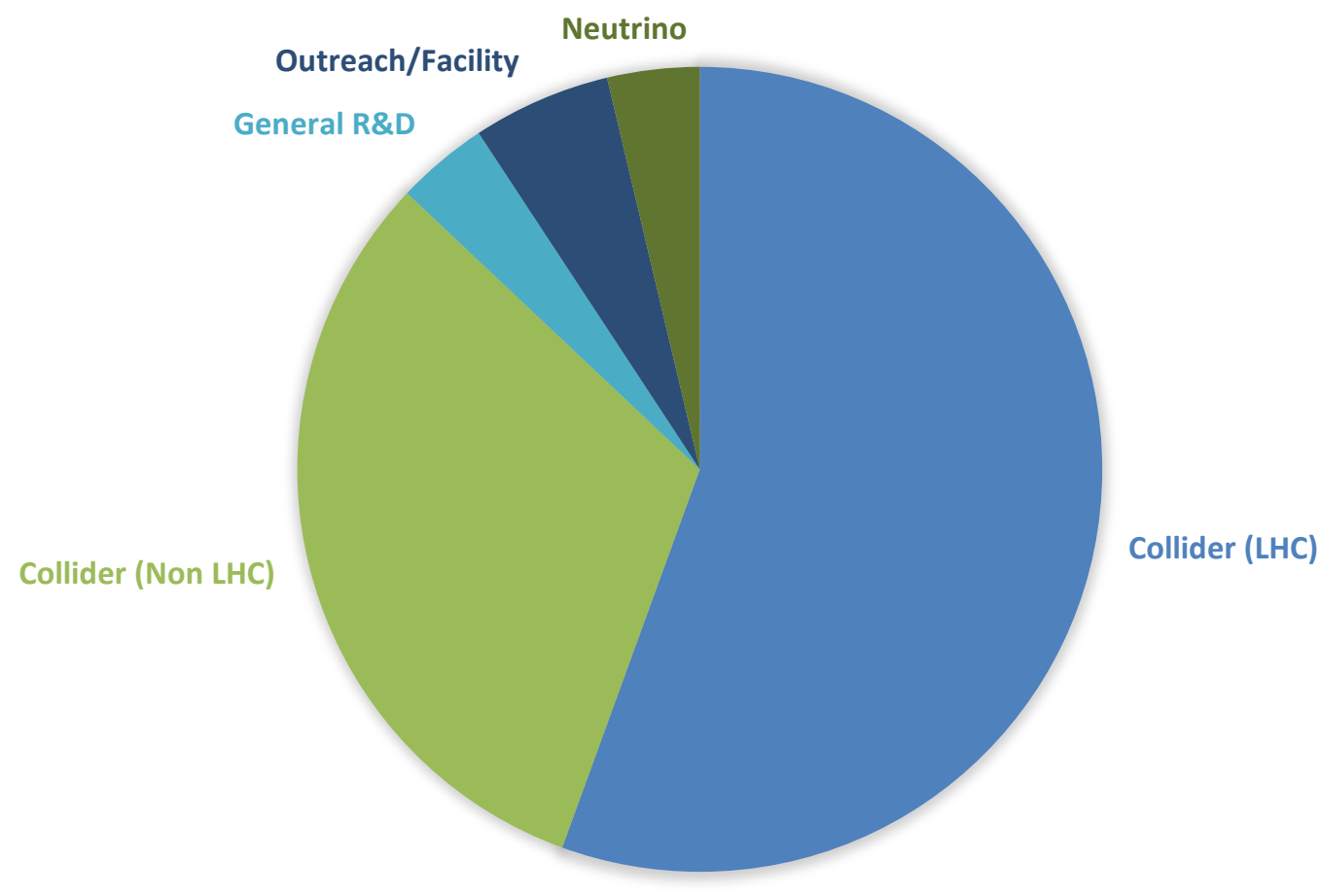

Figure TB-2: The research focus of the different groups that came to the test beam in FY2018.

MicroBooNE report (B.T. Fleming and G.P. Zeller)

MicroBooNE completed its third year of operations in FY 2018 and to date has collected a total of $9.6 \times 10^{20}$ POT $\left(9.2 \times 10^{20}\right.$ POT recorded) of Booster Neutrino beam (BNB) with the detector operational (Figure M-1). Combined detector and DAQ uptime for the year has been $>95 \%$ and the experiment has been averaging $\sim 3 \times 10^{20} \mathrm{POT} /$ year from the BNB due to the excellent performance of the Fermilab accelerator complex. In FY 2018, MicroBooNE operations were handled by series of 5 Run Coordinators from Fermilab, MIT, University of Manchester, and Syracuse University. Shifts were performed across several shift-taking sites including ROC-W at Fermilab and 10 remote shift centers in the U.S., U.K, and Switzerland. 


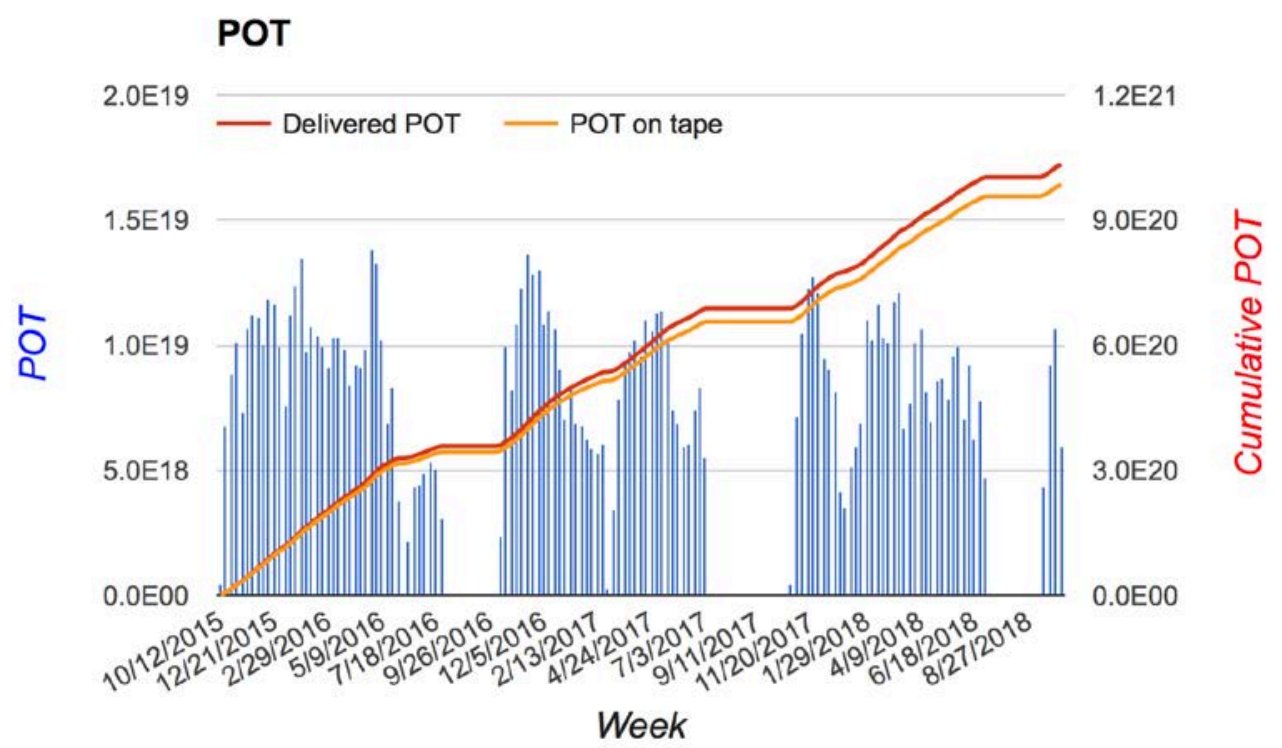

Figure M-1: Total protons on target delivered (red) and recorded (orange) by MicroBooNE since the start of operations in October 2015.

The MicroBooNE detector ran extremely well in 2018 and became the longest running liquid argon TPC to date. In 2018, the experiment finished a complete replacement of the MicroBooNE DAQ system to allow for continued data-taking with all of the most critical machines now back under full service warranty. The collaboration also produced a comprehensive document on lessons learned from MicroBooNE commissioning as a resource for the single phase protoDUNE detector. In early 2018, MicroBooNE generated its first Monte Carlo using data-based calibrations as input and with a complete suite of systematic uncertainties spanning the Booster neutrino flux predictions, modeling of neutrino cross sections on argon, and simulation of detector effects in liquid argon. Given the very large amount of information coming off of liquid argon detectors and the resultantly large file sizes, MicroBooNE also successfully pioneered a significant reduction in data and Monte Carlo file sizes to help speed up file processing. A factor of two improvement in performance was achieved. This experience will be directly applicable to protoDUNE, ICARUS, SBND, and DUNE which will have equally large data volumes. In the spring, MicroBooNE also un-blinded several new data sets including a sample of muon neutrino charged current inclusive and neutral pion events.

MicroBooNE had a record year in 2018 having produced 10 new public notes (http://microboone.fnal.gov/public-notes/) and 5 papers. Among these results, MicroBooNE released its first physics which included 3 neutrino cross section measurements: (1) a measurement of charged track multiplicities in neutrino-nucleus scattering including the first comprehensive test of the GENIE event generator's performance in modeling argon, (https://arxiv.org/abs/1805.06887), (2) the first-ever measurement of the charged current neutral pion cross section in argon (http://microboone.fnal.gov/wp-content/uploads/MICROBOONENOTE-1032-PUB.pdf), and (3) a measurement of single differential cross sections for inclusive charged current muon neutrino scattering in argon (http://microboone.fnal.gov/wpcontent/uploads/MICROBOONE-NOTE-1045-PUB.pdf). These results included a full assessment of systematics. This is the first time such neutrino cross sections have been quantified in argon at these energies - they are important for DUNE because these measurements are on argon and in the region of DUNE's second oscillation maximum. MicroBooNE presented a Wine 
\& Cheese seminar on these results at Fermilab on June 1, 2018. A few examples of these results are shown in Figure M-2.
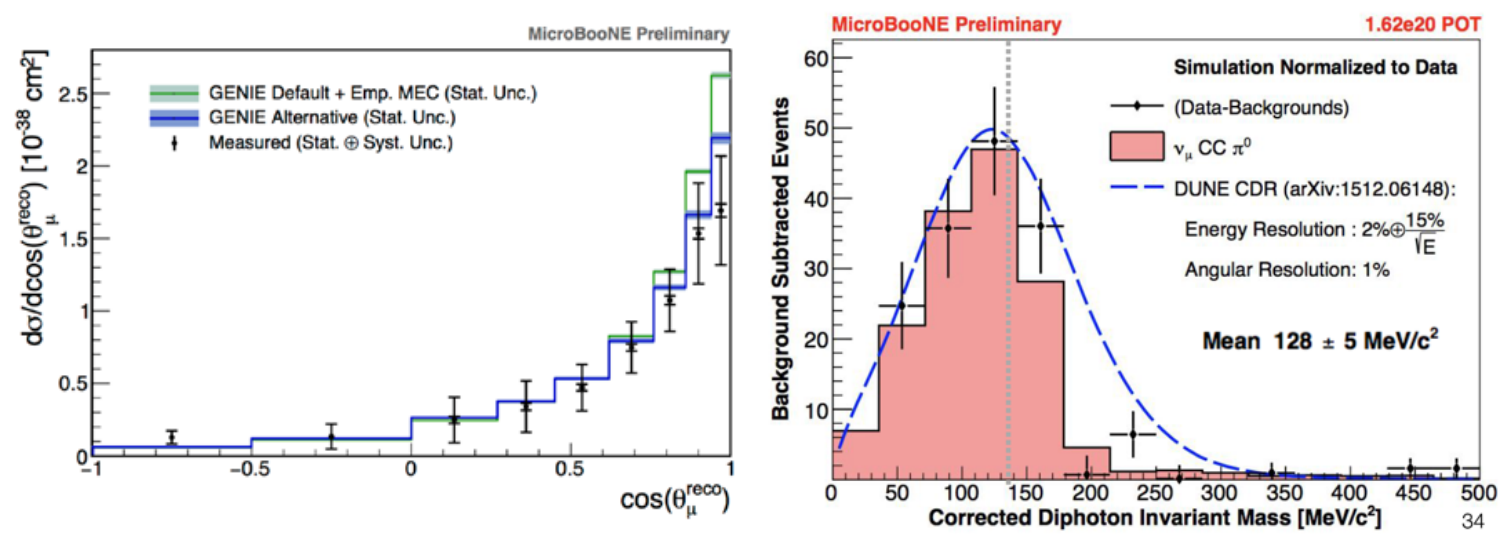

Figure M-2: Left: Measurement of the differential cross section for muon neutrino charged current inclusive interactions on argon in MicroBooNE as a function of muon scattering angle compared to two different GENIE models. As observed in the forward scattering direction, the MicroBooNE data already has some discriminating power between models. Right: Measurement of the di-photon invariant mass spectrum for muon neutrino charged current neutral pion events in MicroBooNE. Overlaid in blue is the distribution that would be achieved with the energy and angular resolutions assumed for DUNE. This measurement meets the physics requirements for DUNE and confirms the power of the technology.

In addition to these pioneering measurements of neutrino-argon scattering, MicroBooNE also published two comprehensive papers on the processing of signals in liquid argon TPCs that appeared in tandem articles in JINST (http://iopscience.iop.org/article/10.1088/17480221/13/07/P07006 and http://iopscience.iop.org/article/10.1088/1748-0221/13/07/P07007). This is the first time that signal processing in a liquid argon TPC has been studied and documented in this level of detail. In 2018, the collaboration also wrote a new paper describing the use of convolutional neural networks to identify electromagnetic activity in liquid argon TPCs that was submitted to Phys. Rev. D. in August 2018 (https://arxiv.org/abs/1808.07269). This analysis represents the first application of convolutional neural networks in the analysis of liquid argon TPC data. A description of this work was also featured in an article in Nature magazine in August 2018 (https://www.nature.com/articles/s41586-018-0361-2.pdf). In summer 2018, MicroBooNE additionally showed progress towards the experiment's signature low energy excess analysis after releasing comparisons of data to simulation for several sideband regions just outside of where MicroBooNE will look for signal events (http://microboone.fnal.gov/wpcontent/uploads/MICROBOONE-NOTE-1038-PUB.pdf). The collaboration also produced its first projected capabilities for constraining the radiative decay rate of the Delta resonance as a check of the background estimates assumed in the MiniBooNE neutrino oscillation analysis (http://microboone.fnal.gov/wp-content/uploads/MICROBOONE-NOTE-1041-PUB.pdf).

E989: Muon g-2 Report (C. Polly and M. Lancaster)

g-2 completed a one-month commissioning run (Run-0) in July 2017 with a beam dominated by protons and recorded 1-20 positrons per fill with 1-4 fills per minute. This was sufficient for a $60 \mathrm{ppm}$ (statistical) precision on g-2. Through to November 2017, substantial upgrades were made to the experiment and the beam delivery systems. This included: shimming of the 
storage ring field, optimizing the g-2 in-ring quadrupoles and kicker, installing infrastructure for cryopumps to improve the storage ring vacuum (SRV), measurements of the kicker pulse with a magnetometer, the installation of the trolley to measure the magnetic field, refurbishment of the collimators, the final straw tracker gas and cooling systems and a study period to improve the SRV by nitrogen purging. AD brought online the delivery ring allowing protons to be removed and a predominantly muon beam to be delivered. 24/7 shifts began on 16-Oct and the shutdown formally ended on 23-Oct. The first beam to the experiment was established on 17-Nov: 0.3E12 POT, with 1-4 bunches per cycle and a cycle every 4-7 seconds. Throughout November and December, a substantial increase in the muons/sec and the positrons/fill was achieved by: M4/M5 tuning, increasing to 8-bunches per cycle, per-bunch kicker timing optimization, inflector current optimization and improvements in the overall operating stability of the experiment. At the start of 2018, a tenfold increase in positrons/fill had been achieved over the commission run: 250e+/fill and the muons/sec rate had increased by a factor of 10,000 to 1,000 muons/sec. Further optimizations in terms of the surface coils and kicker settings were implemented and the first analysis-quality data was taken at the start of February. Data taking was then interrupted shortly after this, when an incident with a cryopump vacuum valve caused damage to the Q1 and Q4 quadrupoles, that required a three-week intervention to diagnose and then repair. The downtime was however used to implement the final upgrades required for production running: the temporal distribution of the beam pulses was optimized and the M2/M3 beamlines were better tuned, additional capacitors in the calorimeter readout were added to reduce the early-time gain changes caused by the beam splash at injection, the stability of the DAQ was improved, the clock-blinding infrastructure was installed, and a kicker discharge pulse system was introduced to reduce sparking, and many double laser-pulse calibration runs were taken. In concert, many simulation studies were performed to better understand the trends observed from the data taken in early February to define the optimum operating conditions for production running. Data taking resumed on 16-March, and on 22-March the accelerator switched to the design 16-pulse operation and this formally ended the Run-1 commissioning period and the data taken after 26-March has defined the "Run-1-Physics" dataset. Throughout this Run-1-Physics period an average of $500 \mathrm{e}+/ \mathrm{fill}$ (50,000 muons/sec) were being recorded which is $50 \%$ of the rate defined in the TDR. The significant evolution in the rate over the past year is shown in Fig. 1 (a) and (b). Over the course of the Run-1Physics period, 5.1e19 POT were delivered: exceeding the base-design set prior to the run by $10 \%$. 


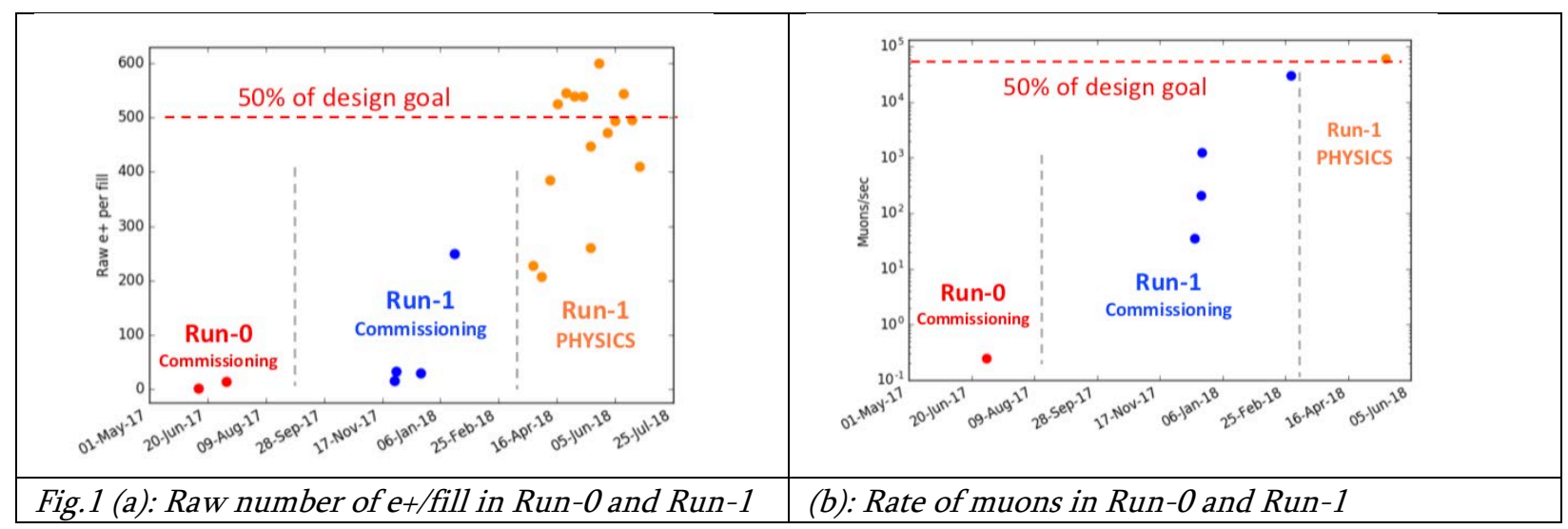

The POT delivered and the raw e+ recorded as a function of time is shown in Fig. 2 (a). The DAQ was live for $90 \%$ of the time when beam was being delivered. The aim of Run- 1 was to record a BNL-size dataset. A dataset almost twice that of the BNL (mu+ and mu-) running was recorded, although the dataset to be used in the final Run-1 analysis will be approximately $\mathrm{x} 1.5$ that of the BNL dataset after data-quality cuts and the need for the data to be taken with consistent operating conditions is considered. This dataset is dominated by the statistical uncertainty: a statistical uncertainty of $0.35 \mathrm{ppm}$ (vs $0.46 \mathrm{ppm}$ at BNL) is expected to be achieved. This in combination with the BNL result would be sufficient to approach a 5 sigma discrepancy with respect to the SM if the FNAL and BNL central values were the same. The dataset has been split into 7 distinct datasets with somewhat different operating conditions (ostensibly different kicker and quadrupole settings). The analysis of one of these datasets, representing approximately $10 \%$ of the data, is at a rather advanced stage. The number of $\mathrm{e}+$ as a function of time in the fill, illustrating the characteristic $\mathrm{g}-2$ "wiggle" is shown for this dataset in Fig 2(b). It has a statistical precision of 1.2ppm.

Fig. 2 (a): Raw number of e+ accumulated over Run-
1 along with the POT delivered.
$\begin{aligned} & \text { (b): Number of e+ as a function of the time in the fill } \\ & \text { for 60hrs of data accumulated in April 2018. The } \\ & \text { frequency of the characteristic “wiggle" is used to } \\ & \text { determine } g \text { - } 2 \text {. }\end{aligned}$

All seven datasets have been processed offline using FermiGrid and further reprocessing incorporating improved reconstruction algorithms, alignments, calibrations and data quality 
cuts is about to begin. The online and detector systems performed very well throughout the run. Data was accumulated at rates up to $250 \mathrm{Mb} / \mathrm{sec}$ by the DAQ system. The field was mapped every 2-3 days using the trolley system and continuously by the fixed probes. In terms of both the field moments and the dipole RMS, the field uniformity is significantly better than at BNL (see Fig.3). The variance in the field has achieved the design goal metrics of being between one-third to one-half that of BNL.

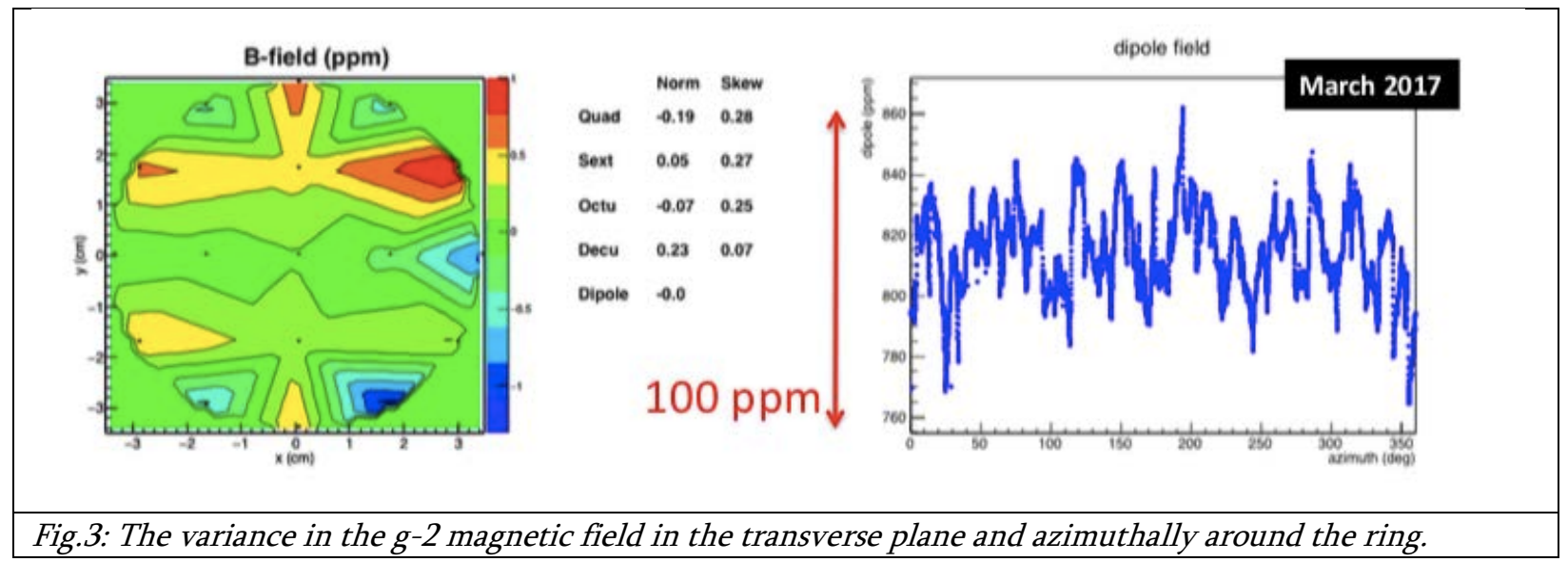

The detector systems: calorimeters, straw-trackers, IBMS and fiber-harps all performed to or better than their design specifications. Some example performance plots illustrating this are shown in Fig.4.

Over the shutdown period a significant body of work has been undertaken to ensure that the experiment can reach its design statistical and systematic goals by the end of FY20. The stored muon flux is presently $50 \%$ of the design goal and the variance in the momentum and spatial distribution of the stored beam is broader than is desirable (see Fig. 5). There is also a significant variance in the hall temperature that affects the calorimeter and field calibrations. 


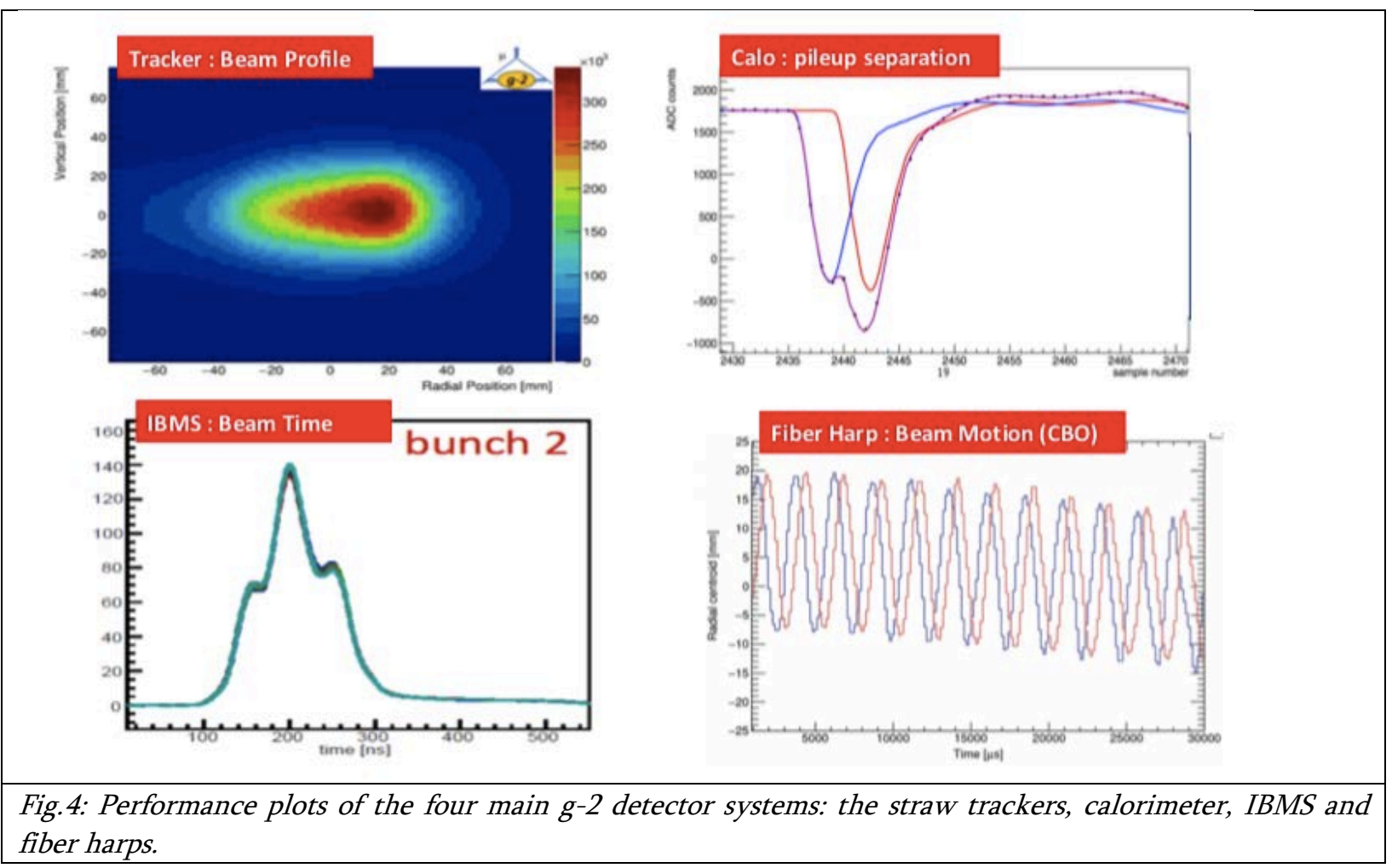

A flux increase of $x 1.5$ is expected to be achieved for the FY19 run by the introduction of momentum collimating wedges in the downstream beamline, and improvements to the g- 2 kicker and quadrupole systems, along with improved reliability in the cryo infrastructure. A further flux increase of $\mathrm{x} 1.4$ will be achieved by the installation of a new open-ended inflector magnet in the 2019 shutdown. This is sufficient to record a dataset of x10 BNL by the end of FY19 and x21 BNL (the design goal) by the end of FY20. The improvements presently being implemented for the kicker system, principally the ability to provide a larger $(60 \mathrm{kV})$ kick, will result in the beam being better centered with a smaller variance. The infrastructure necessary to reduce the hall temperature variations will also be installed prior to the resumption of physics data taking in November 2019.



Fig.5: A comparison of the equilibrium radius of the stored muon beam for the BNL data and for the FNAL data. The FNAL data is offset somewhat from the ideal position and is broader. 


\section{Acknowledgement}

This report gives a brief summary of the performance and output of the accelerator complex and associated accelerator-based experiments during FY 2018. It therefore summarizes the work of many people from Fermilab and from the collaborating institutions. The credit for the successful outcome of the FY 2018 running is shared amongst many. 